BULL. AUSTRAL. MATH. SOC.

$13 B 30,13 E 05$

VOL. $28(1983), 9-12$.

\title{
COMMUTATIVE RINGS WHOSE FACTORS HAVE ARTINIAN RINGS OF QUOTIENTS
}

\author{
WILLIAM D. BLAIR
}

Let $R$ be a commutative ring with unity. Then every factor ring of $R$ has an Artinian total quotient ring if and only if $R$ is a direct sum of one-dimensional Noetherian domains and local Artinian rings .

The problem of characterizing those commutative rings for which every factor ring has some particular finiteness property has been studied for some time. Cohen [2] has shown that those commutative rings enjoying the property that every proper factor ring is an Artinian ring are either onedimensional Noetherian domains or are themselves Artinian. Camillo [1] proved that a commutative ring is Noetherian if and only if every factor ring is a Goldie ring. The purpose of this paper is to consider those commutative rings whose factor rings have an Artinian ring of fractions.

All rings considered here will be commutative rings with unity. Recall that a ring is said to be an order in an Artinian ring if the ring of fractions we obtain when we localize at the set of all non zero-divisors is an Artinian ring. A ring is said to be a Goldie ring if it has no infinite direct sums of ideals and has the ascending chain condition on annihilator ideals.

LEMMA 1. If every factor ring of $R$ is an order in an Artinian ring then $R$ is a Noetherian ring.

Proof. Let $\bar{R}$ be a factor ring of $R$ and let $\bar{R}$ be an order in

Received 7 March 1983. 
$Q$. Since $Q$ is an Artinian ring it satisfies the ascending chain condition on annihilators and this property passes to the subring $\vec{R}$. If $I_{I} \oplus \ldots \oplus I_{n}$ is a direct sum of ideals of $\bar{R}$ then it is easy to see that $I_{1} Q \oplus \ldots \oplus I_{n} Q$ is a direct sum of ideals of $Q$. Since $Q$ has no infinite direct sum of ideals, $\bar{R}$ has no infinite direct sum of ideals. Thus $\bar{R}$ is a Goldie ring. Since every factor ring of $R$ is a Goldie ring we have that $R$ is a Noetherian ring by Camillo's theorem.

The next lemma essentially appears in Small [3].

LEMMA 2. If every factor ring of $R$ is an order in an Artinian ring then each ideal of $R$ has no embedded prime ideals.

Proof. $R$ is a Noetherian ring by Lemma 1 . Let $I$ be an ideal of $R$ and let $P_{1}, \ldots, P_{t}$ denote the associated prime ideals of $I$. Let $\bar{R}=R / I$ and $\bar{P}_{j}=P_{j} / I$ for $j=1, \ldots, t$. Note that $\bar{P}_{1}, \ldots, \bar{P}_{t}$ are the associated prime ideals of zero in $\bar{R}$ and, as such, no $\bar{P}_{j}$ contains a regular element of $\bar{R}$. If $P_{i} \varsubsetneqq P_{k}$ for some $i \neq k$ then $\bar{P}_{i} \varsubsetneqq \bar{P}_{k}$ and if $Q$ denotes the Artinian ring of quotients of $\bar{R}$, then $\bar{P}_{i} Q \varsubsetneqq \bar{P}_{k} Q \varsubsetneqq Q$ since no $\bar{P}_{j}$ contains a regular element. Since $\bar{P}_{i} Q$ and $\bar{P}_{k} Q$ are prime ideals of $Q$, and since all prime ideals of an Artinian ring are maximal, we have a contradiction. Thus $I$ has no embedded primes.

LEMMA 3. Let $R$ be a local Noetherian ring such that each ideal has no embedded primes. If $R$ is not an Artinian ring then $R$ is a onedimensional domain.

Proof. Let $M$ be the maximal ideal of $R$ and suppose that $P$ is a prime ideal such that $P \varsubsetneqq M$. Let $k \in \mathbf{N}$ and consider $I=M^{k} \cap P$. Since $I$ has no embedded primes, we have $I=P$ for otherwise the primary ideal $M^{k}$ would be contained in $P$ and so $M$ would be equal to $P$. Thus $P \subseteq M^{k}$ for all $k \in \mathbb{N}$ and $P \subseteq \bigcap_{k=1}^{\infty} M^{k}$. But $\bigcap_{k=1}^{\infty} M^{k}=(0)$ by the Krull intersection theorem. Hence $R$ is a one-dimensional domain.

LEMMA 4. Let $(0)=Q_{1} \cap \ldots \cap Q_{s}$ be the irredundant decomposition of (0) in a Noetherian ring $R$ in which each ideal has no embedded 
primes. Let $Q_{i}$ be $P_{i}$-primary and suppose that $P_{i}$ is a non-maximal prime $i$ deal for $i=1, \ldots, t$ and that $P_{i}$ is a maximal ideal for $i=t+1, \ldots, s$. Then for $1 \leq i<j \leq s, Q_{i}$ and $Q_{j}$ are comaximal $i$ deals and for $1 \leq i \leq t, Q_{i}=P_{i}$. Furthermore, for $1 \leq i \leq t, R / P_{i}$ is a one-dimensional domain.

Proof. For $1 \leq i<j \leq s$, assume that the maximal ideal $M$ contains both $Q_{i}$ and $Q_{j}$. The localization $R_{M}$ inherits the property that each ideal has no embedded primes and so by Lemma 3 , $Q_{i}=P_{i}=P_{j}=Q_{j}$ or one of $P_{i}$ or $P_{j}$ is $M$, in which case we have a containment relation between $P_{i}$ and $P_{j}$. In either case we have a contradiction and so $Q_{i}$ and $Q_{j}$ are comaximal. If $i \leq t$ then $P_{i} \subseteq M$ for some maximal ideal $M$ and again Lemma 3 implies that $P_{i} R_{M}$ is the zero ideal in $R_{M}$ and so $P_{i}=Q_{i}$, and $R / P_{i}$ is a one-dimensional domain.

We now have the necessary ingredients for our main result.

THEOREM. Let $R$ be a commutative ring. Then every factor ring of $R$ is an order in an Artinian ring if and only if $R$ is a finite direct sum of one-dimensional Noetherian domains and Artinian local rings.

Proof. Since a proper factor ring of a one-dimensional Noetherian domain is Artinian, and since the ring of quotients of a direct sum is the direct sum of the rings of quotients of each factor, it follows that every factor ring of a direct sum of one-dimensional Noetherian domains and Artinian rings is a ring of this form, and thus is an order in an Artinian ring.

Conversely, suppose that every factor ring of $R$ is an order in an Artinian ring. By Lemma $l, R$ is a Noetherian ring and each ideal of $R$ has no embedded primes by Lemma 2 . We apply Lemma 4 to obtain a primary decomposition of the zero ideal: $(0)=P_{1} \cap \ldots \cap P_{s} \cap Q_{1} \cap \ldots \cap Q_{t}$, where each $P_{i}$ is a non-maximal prime ideal and each $Q_{j}$ is a primary ideal whose associated prime ideal is maximal. (Either $s$ or $t$ may be 0. .) In this decomposition each pair of ideals is comaximal and $R / P_{i}$ is 
a one-dimensional domain for $i=1, \ldots, s$. Thus

$$
R \simeq R / P_{1} \oplus \ldots \oplus R / P_{s} \oplus R / Q_{1} \oplus \ldots \oplus R / Q_{t}
$$

where each $R / P_{i}$ is a one-dimensional domain and each $R / Q_{j}$ is a local Artinian ring.

We observe that if $R$ is a ring for which every proper factor ring is an order in an Artinian ring then $R$ may not itself be an order in an Artinian ring. An example of such a ring, due to Kaplansky, appears in Smal I [3].

EXAMPLE. Let $F$ be a field and consider the power series ring $F[[x, y]]$ in two indeterminants. Set $R=F[[x, y]] /\left(x^{2}, x y\right)$. Then $R$ is its own ring of quotients but $R$ is not Artinian. Every proper factor ring of $R$ is itself Artinian except $R / I$ where $I=(x) /\left(x^{2}, x y\right)$. But $R / I \simeq E[[x, y]]$ which is a domain and so $R / I$ does have an Artinian ring of quotients.

\section{References}

[1] Victor P. Camillo, "Commutative rings whose quotients are Goldie", GLasgow Math. J. 16 (1975), 32-33.

[2] I.S. Cohen, "Commutative rings with restricted minimum condition", Duke Math. J. 17 (1950), 27-42.

[3] Lance W. Small, "Orders in Artinian rings", J. Algebra 4 (1966), $13-41$.

Department of Mathematical Sciences, Northern Illinois University,

De Kalb,

ll1inois 60115, USA. 